ISSN 1409-2441

www.ucr.ac.cr
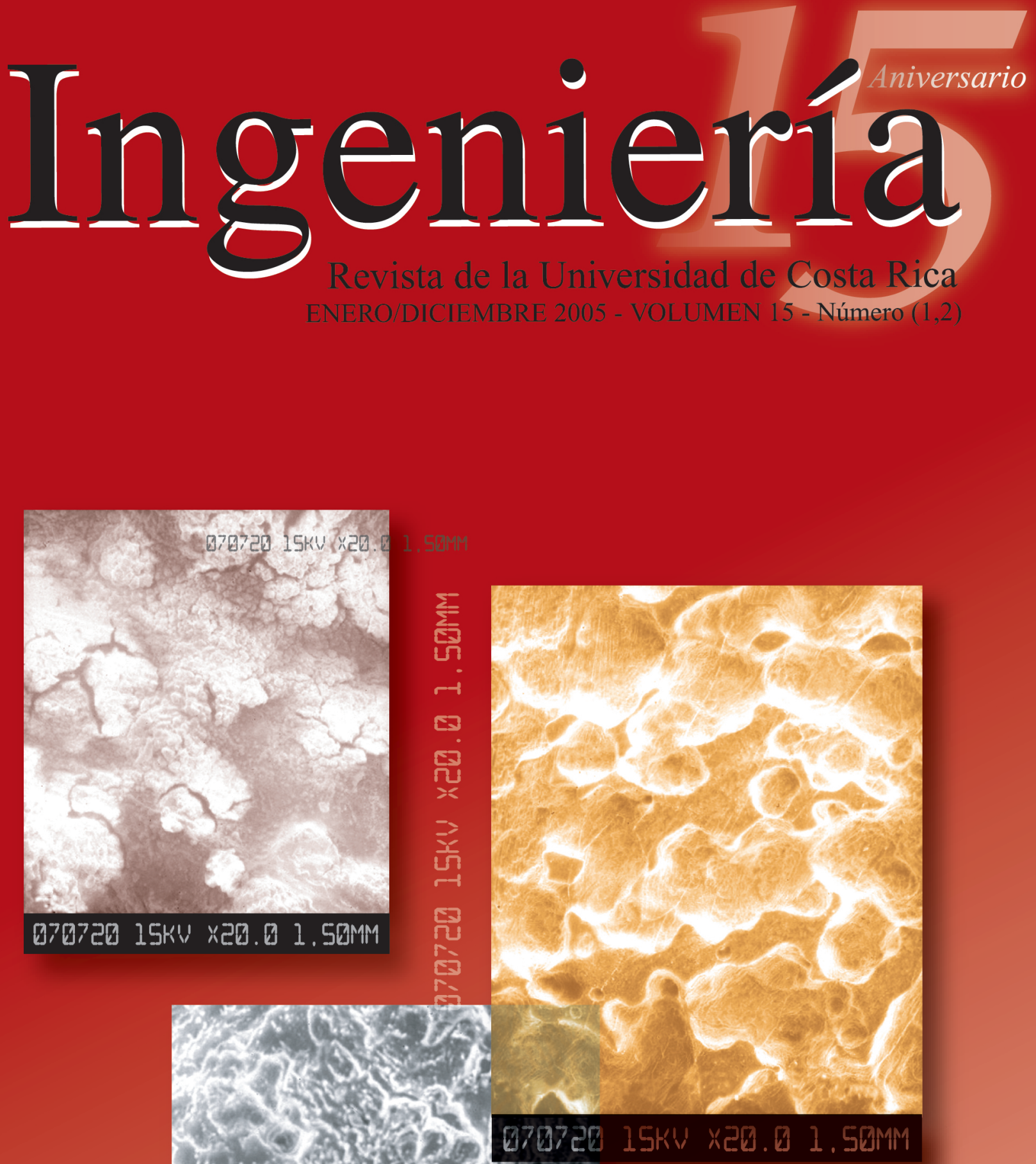

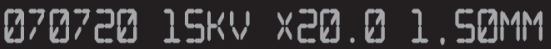

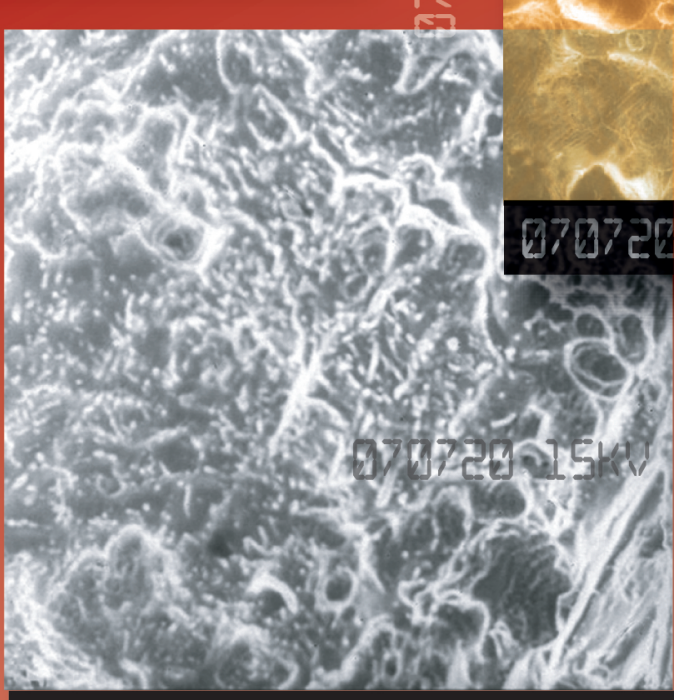

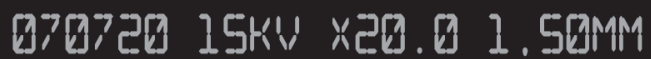




\title{
EVALUACIÓN DE PARÁMETROS CINÉTICOS PARA LA Sacharomyces cerevisiae UTILIZANDO AGUA DE COCO COMO SUSTRATO

\author{
Oscar Ramírez Sánchez,
} Manuel Molina Córdoba
}

\section{Resumen}

Se utilizó el agua de coco (Cocus nucifera), como sustrato para la reproducción de la Sacharomyces cerevisiae; se determinaron las condiciones más adecuadas para el crecimiento de la levadura Sacharomyces cerevisiae, tanto para un proceso "batch" como para un sistema continuo y se obtuvieron algunos parámetros cinéticos. Se encontró que el flujo de aire fue la única de las variables estudiadas que afecta el rendimiento de la producción de biomasa. El pH, la temperatura, la fuente de nitrógeno y la concentración de la fuente de nitrógeno, no tuvieron un efecto significativo para los niveles analizados. Para un sistema "batch" de 2 L, se encontró que al aumentar la concentración de levaduras de (50 a 70) millones de células por mililitro, a una temperatura de $28^{\circ} \mathrm{C}$, se logra reducir la zona "lag" en una hora, y que la velocidad específica máxima de crecimiento es de $0,38 \mathrm{~h}_{-1}$, la constante de saturación de $1,35 \mathrm{~g} / \mathrm{L}$, el rendimiento de 1,68 y la velocidad específica del producto de $0,23 \mathrm{~g} / \mathrm{g} \cdot \mathrm{h}$. Para un sistema continuo, se obtuvo una tasa de dilución máxima de $0,19 \mathrm{~h}_{-1}$ y una tasa de dilución crítica de $0,30 \mathrm{~h}_{-1}$. El proceso de reproducción en dicho sustrato origina una disminución del $41 \%$ del DQO inicial.

Palabras clave: Biomasa, desarrollo sostenible, reproducción, Sacharomyces cerevisiae, coco, cinética, Cocus nucifera

\begin{abstract}
We studied coconut water from Cocus nucifera as substrate for Sacharomyces cerevisiae growth. Temperature, nitrogen source and concentration, and $\mathrm{pH}$ did not have any significant effect on growth; the air flow rate was the only variable that influenced biomass yield. Batch experiments gave a maximum specific growth rate of $0,8 \mathrm{~h}_{-1}$, a saturation constant of $1,35 \mathrm{~g} / \mathrm{L}$, a biomass/substrate yield of 1,68 , a specific product rate of $0,23 \mathrm{~g} / \mathrm{g} \cdot \mathrm{h}$ and by changing inoculum's concentration from (50 to 70) million cell per milliliter lag phase decreased by one hour. In continuous mode, the maximum dilution rate was $0,19 \mathrm{~h}_{-1}$ and the critical dilution rate was $0,30 \mathrm{~h}_{-1}$. Yeast-free broth had a chemical oxygen demand (DQO) $41 \%$ lower than initial coconut water.
\end{abstract}

Key words: Biomass, Sacharomyces cerevisiae reproduction, coconut, kinetic, Cocus nucifera.

Recibido: 25 de febrero del 2005 / Aprobado: 02 de junio del 2005

\section{INTRODUCCIÓN}

El fruto del cocotero (Cocus nucifera), es explotado comercialmente para aprovechar la cáscara (exocarpio y mesocarpio) y la nuez (endocarpio), a fin de obtener una serie de productos de interés comercial como el aceite, la pulpa deshidratada, etc. El agua contenida en la nuez, constituye actualmente un subproducto que no es utilizado por las industrias y es eliminado de los procesos, sin ningún tipo de tratamiento que permita reducir los problemas de contaminación ambiental.
Algunos trabajos (Rodríguez, 1987; Vargas, 1992) muestran que el fruto está constituido por un $35 \%$ de cáscara, $60 \%$ de pulpa y un $8 \%$ de agua.

El agua de coco contiene sustancias como azúcares, proteínas, minerales y vitaminas, que la convierten en un sustrato potencialmente bueno para la producción de biomasa o para otros fines. Algunos elementos trazas, encontrados en el agua de coco son: potasio, $312 \mathrm{mg} / \mathrm{mL}$; fósforo $37 \mathrm{mg} /$ $\mathrm{mL}$; sodio $105 \mathrm{mg} / \mathrm{mL}$ y calcio $29 \mathrm{mg} / \mathrm{mL}$ (Palasi, 1952; Stanier, 1986). 
Se determinaron las condiciones más adecuadas para el crecimiento de la levadura Sacharomyces cerevisiae, tanto para un proceso "batch" como para un sistema continuo y se obtuvieron algunos parámetros cinéticos.

\subsection{Cinética de crecimiento}

El crecimiento microbiano fue estudiado y modelado por Monod para un sistema discontinuo. (Amato, 1992; Fogler, 2001; Jorgensen, 1959; Palasi, 1952; Prescott \& Dunns, 1983; Quintero, 1981). La ecuación de Monod, relaciona la velocidad máxima de crecimiento con la concentración del sustrato limitante, la que se expresa como:

$\mu=\mu_{\max }\left(\frac{S}{K s+S}\right)$

Donde:

$\mu$ : velocidad específica de crecimiento, $\left(\mathrm{h}^{-1}\right)$

$\underset{\left(\mathrm{h}^{-1}\right)}{\mu_{\max }}$ : velocidad específica máxima de crecimiento,

$S$ : concentración de sustrato limitante, $(\mathrm{g} / \mathrm{L})$

$K s$ : constante de saturación, (g/L).

El valor $\mu_{\max }$ se obtiene a partir de la pendiente máxima de la curva de crecimiento del microorganismo y la constante de saturación $K s \quad(\mathrm{~g} / \mathrm{L})$, es aproximadamente igual a la concentración de azúcares totales en el medio de cultivo para la fase estacionaria de crecimiento del microorganismo.

\subsection{Cultivo continuo}

Una de las técnicas básicas de cultivo continuo es el uso de un quimiostato, caracterizado porque la suspensión de biomasa está perfectamente mezclada y opera en estado estacionario; su importancia radica en la posibilidad de fijar la velocidad específica de crecimiento, entre cero y un valor máximo, para un determinado tipo de sustrato (Amato, 1992; Galindo, 1993; Palasi, 1952; Pescott \& Dunns,1984).
El tiempo de retención $\mathrm{t}_{\mathrm{r}}$, está dado por $\mathrm{V} / \mathrm{F}$, donde $\mathrm{V}$ es el volumen del reactor y $\mathrm{F}$ es la velocidad de flujo volumétrico del sustrato y células, cuya concentración es So. La tasa de dilución, $D=F / V$, representa una variable esencial de operación en un sistema continuo.

El aumento en la biomasa está dado por el siguiente balance:

$\mathrm{VdX}=\mathrm{V} \mu_{\max } \mathrm{X} d t-F X d t$

Dividiendo entre V y dt:

$\mathrm{dX} / \mathrm{dt}=\left(\mu_{\max }-\mathrm{D}\right) \mathrm{X}$

en condiciones de estado estacionario, se obtiene que:

$\mu=\mathrm{D}$

De un balance para el sustrato limitante se tiene que:

$\mathrm{dS} / \mathrm{dt}=\mathrm{DSo}-\mathrm{DS}-1 / \mathrm{Y} \mu \mathrm{X}$

siendo Y, la constante de conversión o rendimiento que describe la relación entre la biomasa producida y el sustrato:

$\mathrm{dX} / \mathrm{dt}=-\mathrm{Y} \mathrm{dS} / \mathrm{dt}$

En estado estacionario, $\mathrm{d} S / \mathrm{d} t=0, \mathrm{y}$ con la ecuación 4 se obtiene:

$\left(\mathrm{S}_{\mathrm{O}}-\mathrm{S}\right)=\mathrm{X} / \mathrm{Y}$

sustituyendo en la ecuación 1 , se obtienen las expresiones para $S$ y $X$, para un régimen permanente:

$$
\begin{aligned}
& S=\frac{K_{s} D}{\left(\mu_{\max }-D\right)} \\
& X=Y\left(S o-\frac{K_{s} D}{\mu_{\max }-D}\right)
\end{aligned}
$$


Las ecuaciones (8) y (9), permiten predecir las concentraciones de la masa celular y del sustrato,en el reactor, para un valor específico de la tasa de dilución, si se conocen los valores de las constantes $\mu_{\max }, K s$ y $Y$ del microorganismo utilizado. De no conocerse dichos valores, se determinan en un cultivo "batch".

La dilución crítica "wash out", se obtiene cuando la concentración de sustrato en el medio y la inicial son iguales, esto es: $S=S o$. Sustituyendo este valor nuevamente en la ecuación (1), se obtiene:

$\mu=D c r i=\mu_{\max }\left(\frac{S}{K s+S}\right)$

valor máximo que puede obtenerse para un factor de dilución $D$.

La producción de biomasa por unidad de volumen por unidad de tiempo del cultivo se denomina productividad, $P$, que está dada por:

$\mathrm{P}=\mathrm{DX}$

El valor máximo de biomasa, está dada por:

$D_{\max }=\mu_{\max }\{1-\sqrt{K s /(K s+S o)}\}$

sustituyendo la ecuación (12) en la ecuación (9), se obtiene:

$X_{\max }=Y[S o+K s-\sqrt{K s(K s+S o}]$

\subsection{Requerimientos nutricionales y ambientales}

Las levaduras requieren de condiciones ambientales y nutricionales adecuadas para su crecimiento y reproducción. Algunos elementos esenciales son: carbono, nitrógeno, fósforo, calcio, hierro, potasio, hidrógeno, oxígeno, azufre y magnesio. Las vitaminas y otros compuestos orgánicos son también necesarios para el desarrollo satisfactorio y el funcionamiento normal de muchos tipos de levaduras, como la S. cerevisae (Jorgensen, 1959; Quintero, 1981; Rodríguez, 1987: Vong Tosi, 1996).
El potasio resulta indispensable para el crecimiento de las levaduras en cantidades relativamente pequeñas $\left(10^{-2}\right.$ a $\left.10^{-4}\right) \mathrm{mol} / \mathrm{L}$. El calcio y el magnesio forman parte de los cofactores y de la pared celular, siendo el magnesio fundamental para la estabilidad del ATP y para la formación de enzimas; se le emplea a una concentración de $0,25 \mathrm{~g} / \mathrm{L}$ (Quintero, 1981; Stanier, 1986).

La tiamina muchas veces acelera la tasa de fermentación. $S$. cerevisae requiere de biotina en cantidades de 0,75 ppm a 2,5 ppm. La biotina participa en la fijación del $\mathrm{CO}_{2}$ y en la síntesis de ácidos grasos (Palasi, 1952; Stanier, 1986).

Los parámetros óptimos para el crecimiento celular, desde el punto de vista de operación, son según (Vargas, 1992):

Temperatura: el ámbito para el crecimiento de las levaduras se encuentra entre $25^{\circ} \mathrm{C}$ a $30{ }^{\circ} \mathrm{C}$.

pH: las levaduras se ven favorecidas por un $\mathrm{pH}$ próximo a 4 ó 4,5.

Oxígeno: la cantidad de oxígeno necesaria es de al menos $1 \mathrm{~g} \mathrm{O} / \mathrm{g}$ de levadura (Amato, 1992; Quintero, 1981).

\section{METODOLOGÍA, MATERIALES Y EQUIPO EXPERIMENTAL}

\subsection{Equipo}

Bioreactor: se utilizó un bioreactor de $5 \mathrm{~L}$, con los puertos necesarios para la toma de muestras, sistemas de enfriamiento y/o calentamiento, sensores de oxígeno y $\mathrm{pH}$; este equipo se utilizó para las pruebas de seguimiento cinético. Las pruebas para encontrar el efecto de cada una de las variables de diseño, se realizaron en erlemmeyers de $250 \mathrm{~mL}$, controlando la temperatura mediante el uso de un baño con termostato $\left( \pm 1{ }^{\circ} \mathrm{C}\right)$.

Recuentodemicroorganismos: laconcentración de microorganismos se realiza utilizando una cámara Neubauer de un área de $0,0025 \mathrm{~mm}^{2}$ 
y una profundidad de $0,1 \mathrm{~mm}$, junto con un microscopio Southern Precision.

Sustrato: se recolectó agua de coco fresca durante el procesamiento del fruto, en una empresa dedicada a la industrialización de la pulpa de coco. Esta se transportó de inmediato, fue envasada en erlenmeyers de un litro, los que fueron debidamente tapados y se esterilizaron durante 20 min a una presión de $103,4 \mathrm{kPa}(15$ psig), para asegurar su conservación durante toda la fase experimental y disponer de una única materia prima.

Nutrientes: el sustrato se enriqueció con elementos como zinc, magnesio, potasio y tiamina, estos se muestran en el Cuadro 1.

Cuadro 1. Nutrientes adicionados en la formulación del medio de cultivo

Compuesto

$\begin{array}{lc}\text { Fosfato de amonio } & 0,15 \\ \text { Fosfato de potasio monobásico } & 0,15 \\ \text { Sulfato de magnesio } & 0,12 \\ \text { Sulfato de zinc } & 0,10 \\ \text { Extracto de levadura } & 0,10 \\ \text { Tiamina } & 0,005\end{array}$

Fuente:(Los autores)

Levadura: se utilizó Sacharomyces cerevisiae comercial deshidratada; se rehidrató antes de utilizarla a una temperatura de $28{ }^{\circ} \mathrm{C}$.

Azúcares totales: se determinaron utilizando el método de la antrona, las lecturas se realizaron en un espectrofotómetro Jenway, modelo 6105, a una longitud de onda de $665 \mathrm{~nm}$; las determinaciones se realizaron por triplicado.

Determinación del DQO: se determinó el DQO antes y después del proceso de reproducción de la levadura, mediante el método del dicromato de potasio, para cuantificar el efecto en la carga biológica, se siguió el método descrito por (Metcalf L.\& Eddy H.,1995).

\subsection{Condiciones para el crecimiento de la Sacharomyces cerevisiae}

Con el objetivo de estudiar las condiciones para un adecuado crecimiento de la $S$. cerevisiae, se diseñó un experimento de tipo factorial, bloqueado con cuatro variables de mayor influencia en el crecimiento microbiano a dos niveles (Ramírez, 1994; Vong Tosí, 1996).

Variables fijas: tipo de ácido, el $\mathrm{HCl}$; el nivel de agitación se estableció en $90 \mathrm{rpm}$; la concentración inicial de levadura en cuarenta millones de células por mililitro; el tiempo del experimento en $30 \mathrm{~h}$ y la temperatura en $28^{\circ} \mathrm{C}$.

\section{Variables de diseño:}

pH: 4 y 4,5 .

Fuente de nitrógeno: urea y nitrato de amonio, ambas fuentes en concentraciones equivalentes. Las concentraciones utilizadas fueron $0,75 \mathrm{~g} / \mathrm{L}$ de urea y $1,25 \mathrm{~g} / \mathrm{L}$ de nitrato de amonio.

Flujo de aire: se utilizaron los valores de $35 \mathrm{~mL} / \mathrm{s}$ y $65 \mathrm{~mL} / \mathrm{s}$.

Variable de respuesta: el rendimiento $(Y)$, expresado como la razón entre la producción de biomasa y el consumo de sustrato o azúcares totales.

Se utilizó un baño con control de temperatura, provisto de agitación, con capacidad para nueve erlenmeyers de $250 \mathrm{~mL}$, sujetos por abrazaderas.

Se prepararon $20 \mathrm{~mL}$ de inóculo de una determinada concentración de levadura y se agregaron a $180 \mathrm{~mL}$ de medio de cultivo, con una concentración conocida de azúcares. Para cada una de las pruebas experimentales se procedió de la siguiente forma:

Se preparó el inóculo, se tomaron en un erlenmeyer $400 \mathrm{~L}$ del medio de cultivo y se agregaron 0,016 g de levadura, se mantuvo a $30^{\circ} \mathrm{C}$ por $30 \mathrm{~min}$, con agitación. 
El medio de cultivo se dividió en dos partes iguales y se reguló el pH a cada una de las partes, de acuerdo con los niveles de estudio.

En cada erlenmeyer de $250 \mathrm{~mL}$, se agregaron 180 mL del medio de cultivo de composición conocida (Cuadro 2) y se ajustaron las condiciones de la fuente de nitrógeno, colocando a cada erlenmeyer un tapón de algodón y se recubrieron con papel aluminio.

Se autoclavaron los erlenmeyers para esterilizar los medios de cultivo y posteriormente enfriarlos; se colocaron los erlenmeyers en el baño de temperatura controlada y se agregaron $20 \mathrm{~mL}$ de inóculo de concentración conocida de levadura.

\subsection{Seguimiento cinético}

Se obtuvo la cinética del crecimiento de la $S$ cerevisiae. El crecimiento del microorganismo se llevó a cabo bajo condiciones fijas, usando la urea como fuente de nitrógeno. Las pruebas del seguimiento cinético se realizaron utilizando un bioreactor de cinco litros tipo "batch", agregando cuatro litros de medio de cultivo e inoculando con la levadura; se tomaron muestras de cinco mililitros cada hora para medir concentración de levaduras y consumo de azúcares; la temperatura se mantuvo constante a $28{ }^{\circ} \mathrm{C}$. Además se monitoreó el porcentaje de oxígeno disuelto, el $\mathrm{pH}$ y la temperatura.

En una segunda repetición se incrementó la concentración inicial de levaduras de (50 a 70) millones de células $/ \mathrm{mL}$ para disminuir la duración de la fase de letargo, el seguimiento se realizó cada hora hasta alcanzar la fase estacionaria.

\subsection{Cultivo continuo}

Se implantó el quimiostato, mostrado en la Figura 1, el cual consiste de un "tanque agitado" con una suspensión de biomasa perfectamente mezclada, la cual se alimenta con un cultivo fresco, a una tasa constante.

\subsection{Determinación de la tasa de dilución}

El equipo empleado es el mismo de la etapa anterior, con la variación del uso de un volumen de reacción de $2 \mathrm{~L}$ y un inóculo con una concentración de 70 millones de células de levadura $/ \mathrm{mL}$. Este equipo, en el arranque se opera como un sistema "batch", hasta que transcurre una hora; luego se determinan las concentraciones de biomasa y de sustrato, a partir de aquí, se inicia el proceso continuo.

Se toman muestras a intervalos regulares de una hora para monitorear las concentraciones de biomasa, sustrato, oxígeno disuelto y $\mathrm{pH}$, hasta que se alcanza la operación en estado estacionario. El proceso anterior se repite nuevamente para diferentes flujos de alimentación, logrando obtener la variación de la concentración de biomasa y sustrato con el flujo de alimentación, que a su vez se relacionan con la tasa de dilución, $D$.

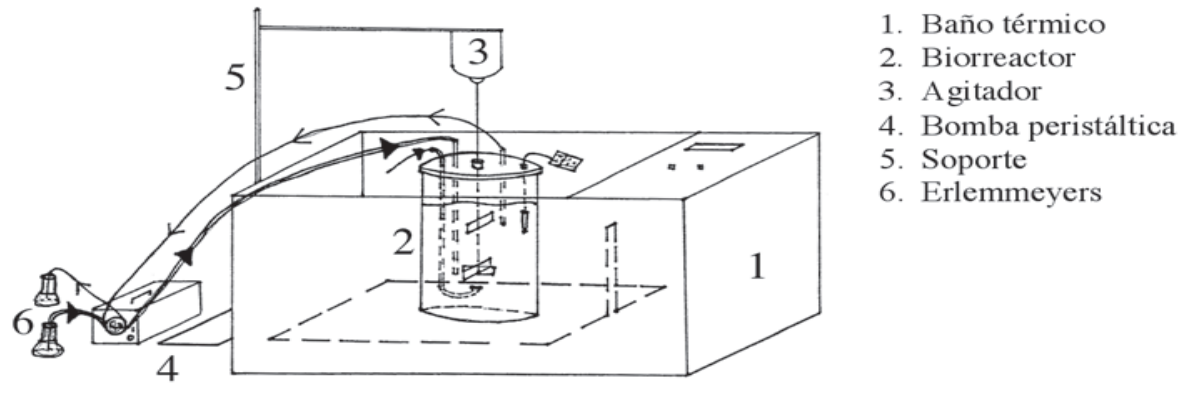

Figura 1. Esquema del quimiostato para pruebas continuas. 


\subsection{Caracterización del agua de coco}

En el Cuadro 2 se presenta la caracterización del agua de coco utilizada.

Cuadro 2. Composición del agua de coco*

\begin{tabular}{|c|c|}
\hline Variable & Valor \\
\hline $\mathrm{pH}$ & 6,69 \\
\hline Densidad, $\rho\left(\mathrm{kg} / \mathrm{m}^{3}\right)$ & 1017 \\
\hline Azúcares totales, $\mathrm{S}_{\mathrm{T}},(\mathrm{g} / \mathrm{L})$ & 4,673 \\
\hline Azúcares reductores, $\mathrm{S}_{\mathrm{R}},(\mathrm{g} / \mathrm{L})$ & 1,50 \\
\hline Sólidos totales $\mathrm{C}_{\mathrm{ST}},(\mathrm{mg} / \mathrm{mL})$ & 54,10 \\
\hline Sólidos solubles, $\mathrm{C}_{\mathrm{SS}},(\mathrm{mg} / \mathrm{mL})$ & 47,02 \\
\hline Sólidos insolubles, $\mathrm{C}_{\mathrm{www}}(\mathrm{mg} / \mathrm{Ml})$ & 3,18 \\
\hline Humedad, H, (\%) & 95,51 \\
\hline
\end{tabular}

* Valores por triplicado

Fuente: (Los autores)

\section{RESULTADOS EXPERIMENTALES}

\subsection{Evaluación de condiciones de operación}

De experimentos previos utilizando como variables: $\mathrm{pH}$, temperatura, fuente de nitrógeno y lujo de aire a los niveles de $13,75 \mathrm{~mL} / \mathrm{s}$ y $56,25 \mathrm{~mL} / \mathrm{s}$, se encontró que solamente esta última variable es significativa y positiva. A partir de dicha información, se utilizaron las condiciones que se muestran en el Cuadro 3, donde, además, se presentan los rendimientos obtenidos y el análisis estadístico. Los experimentos se realizaron a una temperatura de $28{ }^{\circ} \mathrm{C}$ y el rendimiento promedio $(\mathrm{X} / \mathrm{So})$ obtenido fue de 0,896 .

Cuadro 3. Resultados experimentales de la segunda etapa experimental

\begin{tabular}{|c|c|c|c|c|c|}
\hline \multicolumn{3}{|c|}{ (A) Niveles utilizados } & \multicolumn{2}{|c|}{ Iinferior (-) } & Superrior (+) \\
\hline \multicolumn{3}{|l|}{ 1: $\mathrm{pH}$} & \multicolumn{2}{|c|}{4,0} & 4,5 \\
\hline \multicolumn{3}{|c|}{ 2: Fuente de nitrógeno: $\mathrm{F}_{\mathrm{N} 2}$} & \multicolumn{2}{|c|}{ Urea } & Nitrato de amonio \\
\hline \multicolumn{3}{|c|}{ 3: Concentración de nitrógeno: $\mathrm{C}_{\mathrm{N} 2}(\mathrm{~g} / \mathrm{L})$} & \multicolumn{2}{|c|}{0,75} & 1,25 \\
\hline \multicolumn{3}{|c|}{$\begin{array}{l}\text { 4: Flujo de aire: } \mathrm{F}_{\text {aire }}(\mathrm{mL} / \mathrm{s}) \\
\text { (B) DISTRIBUCIÓN DEL DISEÑO }\end{array}$} & \multicolumn{2}{|c|}{35} & 65 \\
\hline Corrida & pH & $\begin{array}{l}\text { Fuente de } \mathbf{N}_{2} \\
\qquad\left(\mathbf{F}_{\mathrm{N} 2}\right)\end{array}$ & $\begin{array}{l}\text { Concentración de } \mathbf{N}_{2} \\
\qquad \begin{array}{c}\left(\mathrm{C}_{\mathrm{N} 2}\right) \\
(\mathrm{g} / \mathrm{L})\end{array}\end{array}$ & $\begin{array}{c}\text { Flujo de aire } \\
\qquad \begin{array}{c}\left(\mathbf{F}_{\text {aire }}\right) \\
(\mathrm{L} / \mathrm{s})\end{array}\end{array}$ & $\begin{array}{c}\text { Rendimiento } \\
\text { (Y) }\end{array}$ \\
\hline 1 & - & - & - & - & 0,654 \\
\hline 2 & + & - & - & - & 0,708 \\
\hline 3 & - & + & - & - & 0,638 \\
\hline 4 & + & + & - & - & 0,711 \\
\hline 5 & - & - & + & - & 0,747 \\
\hline 6 & + & - & + & - & 0,602 \\
\hline 7 & - & + & + & - & 0,662 \\
\hline 8 & + & + & + & - & 0,612 \\
\hline 9 & - & - & - & + & 1,166 \\
\hline 10 & + & - & - & + & 0,992 \\
\hline 11 & - & + & - & + & 1,242 \\
\hline 12 & + & + & - & + & 1,096 \\
\hline 13 & - & - & + & + & 1,153 \\
\hline 14 & + & - & + & + & 0,906 \\
\hline 15 & - & + & + & + & 1,211 \\
\hline 16 & + & + & + & + & 1,234 \\
\hline \multicolumn{6}{|c|}{ ( C ) RESULTADOS ESTADÍSTICOS } \\
\hline \multicolumn{5}{|c|}{ Rendimiento promedio } & 0,896 \\
\hline \multicolumn{5}{|c|}{ Desviación estándar de los efectos e interacciones mayores } & 0,036 \\
\hline \multicolumn{5}{|c|}{ Valor de $t$-student para $5 \mathrm{~g} .1$ con un $95 \%$ de confianza } & 2,571 \\
\hline \multicolumn{5}{|c|}{ Intervalo de no significancia para un $95 \%$ de confianza } & {$[-0,092,+0,092]$} \\
\hline
\end{tabular}

Fuente: (Los autores) 
Cuadro 4. Resultados estadísticos

\begin{tabular}{|c|c|c|c|c|c|}
\hline Efecto & Valor de efecto & Interacción & Valor de interacción & $\begin{array}{l}\text { Interacción } \\
\text { superior }\end{array}$ & $\begin{array}{c}\text { Valor de } \\
\text { interacción } \\
\text { superior }\end{array}$ \\
\hline 1 & $-0,076$ & $1-2$ & 0,052 & $1-2-3$ & 0,040 \\
\hline 2 & 0,060 & $1-3$ & $-0,028$ & $1-2-4$ & 0,023 \\
\hline 3 & $-0,010$ & $1-4$ & $-0,059$ & $1-3-4$ & 0,052 \\
\hline \multirow[t]{3}{*}{4} & 0,4587 & $2-3$ & 0,018 & $2-3-4$ & 0,033 \\
\hline & & $2-4$ & 0,082 & $1-2-3-4$ & 0,020 \\
\hline & & $3-4$ & 0,012 & & \\
\hline
\end{tabular}

En el Cuadro 4 se presentan los efectos e interacciones. Del análisis estadístico se encontró que para el intervalo $[-0,092,+0,092]$ con un nivel de significancia del $95 \%$, solamente el flujo de aire fue significativo y resultó ser positivo, por lo que es deseable utilizar el nivel superior. El valor de $\mathrm{pH}$ puede fijarse en el nivel inferior para que contribuya a inhibir el crecimiento de bacterias, lo que ha sido establecido también por otros autores (Amato, 1992; Jorgensen, 1959; Quintero, 1981). Como fuente de nitrógeno se escogió la urea, por su menor costo y a una concentración de $0,75 \mathrm{~g} /$ $\mathrm{mL}$, puesto que el sustrato aporta nitrógeno.

\subsection{Proceso discontinuo}

Después de fijar las condiciones de operación, se procedió a estudiar el comportamiento cinético de la $S$. cerevisiae y obtener algunos parámetros cinéticos, como la velocidad específica máxima de crecimiento, $\left(\mu_{\text {máx }} \mathrm{h}^{-}\right.$ 1) y la constante de saturación $K s(\mathrm{~g} / \mathrm{L})$. Las curvas de crecimiento para la $S$. cerevisae se muestran en las Figuras 2 y 3.

Analizando la Figura 2, se encontró que la fase de letargo o ajuste del microorganismo al medio de cultivo, tuvo una duración aproximada de 4 h; que la fase de crecimiento exponencial concluyó aproximadamente a las 21 h; que la concentración de biomasa permaneció aproximadamente constante después de dicho tiempo, razón por la que la producción de biomasa puede suspenderse mucho antes. Se tiene la posibilidad de reducir la fase de letargo, mediante el crecimiento de la levadura en un medio semejante o aumentando la concentración inicial de células.
Los resultados también muestran la cantidad de sustrato residual (azúcares) que no fueron metabolizados. Es evidente que esta reducción del sustrato provoca una reducción sustancial del $\mathrm{DQO}$ o DBO, con lo que se reduce el problema de contaminación y consecuentemente, la facilidad de tratar posteriormente estos residuos.

Alalcanzarelestadoestacionario, la concentración de levadura fue de 4,5 g/L. La Figura 3 muestra la curva de crecimiento de la levadura obtenida utilizando una concentración de $S$. cerevisiae de 70 millones por mililitro.

Esta segunda corrida experimental presentó una disminución de una hora en la fase de letargo del microorganismo, con respecto al comportamiento ,cuando se utilizaron 50 millones de células por mililitro; hay un aumento del $40 \%$ en la concentración inicial de levadura en el medio de cultivo. Como resultado, la fase de crecimiento exponencial se dio entre las $3 \mathrm{~h}$ y $15 \mathrm{~h}$, del desarrollo del experimento.

Observando simultáneamente las curvas para la concentración de biomasa y sustrato, se encontró que a las 16 h de operación se inició la fase estacionaria, la concentración de azúcares totales en esta fase fue de aproximadamente de $1,13(\mathrm{~g} / \mathrm{L})$.

En la Figura 4 se presentan los valores promedios para el seguimiento del $\mathrm{pH}$ y el porcentaje de oxígeno disuelto en el medio de cultivo, el pH se mantuvo aproximadamente en un valor de 4. Se observó, además, como el porcentaje de oxígeno disuelto en el medio de cultivo se 


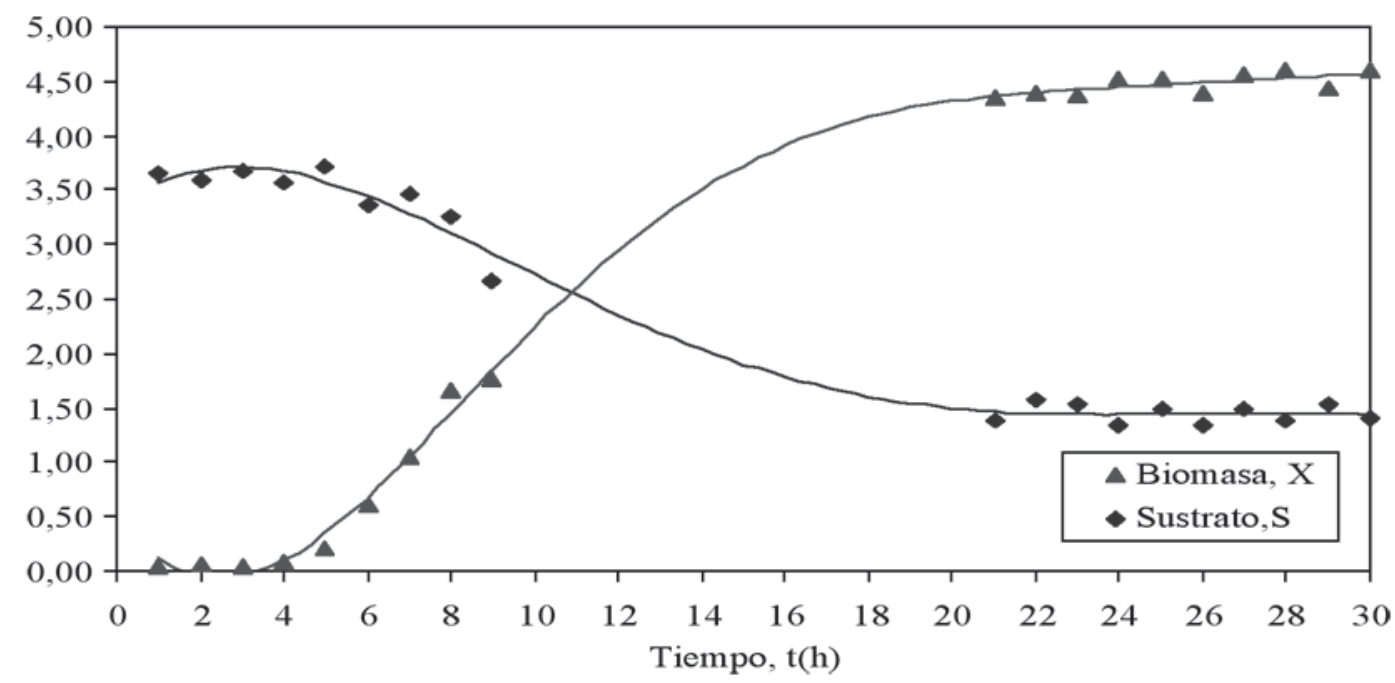

Figura 2. Corrida experimental del seguimiento cinético de la Sacharomyces cerevisiae a una concentración inicial de 50 millones por mililitro.

Fuente: (Los autores)

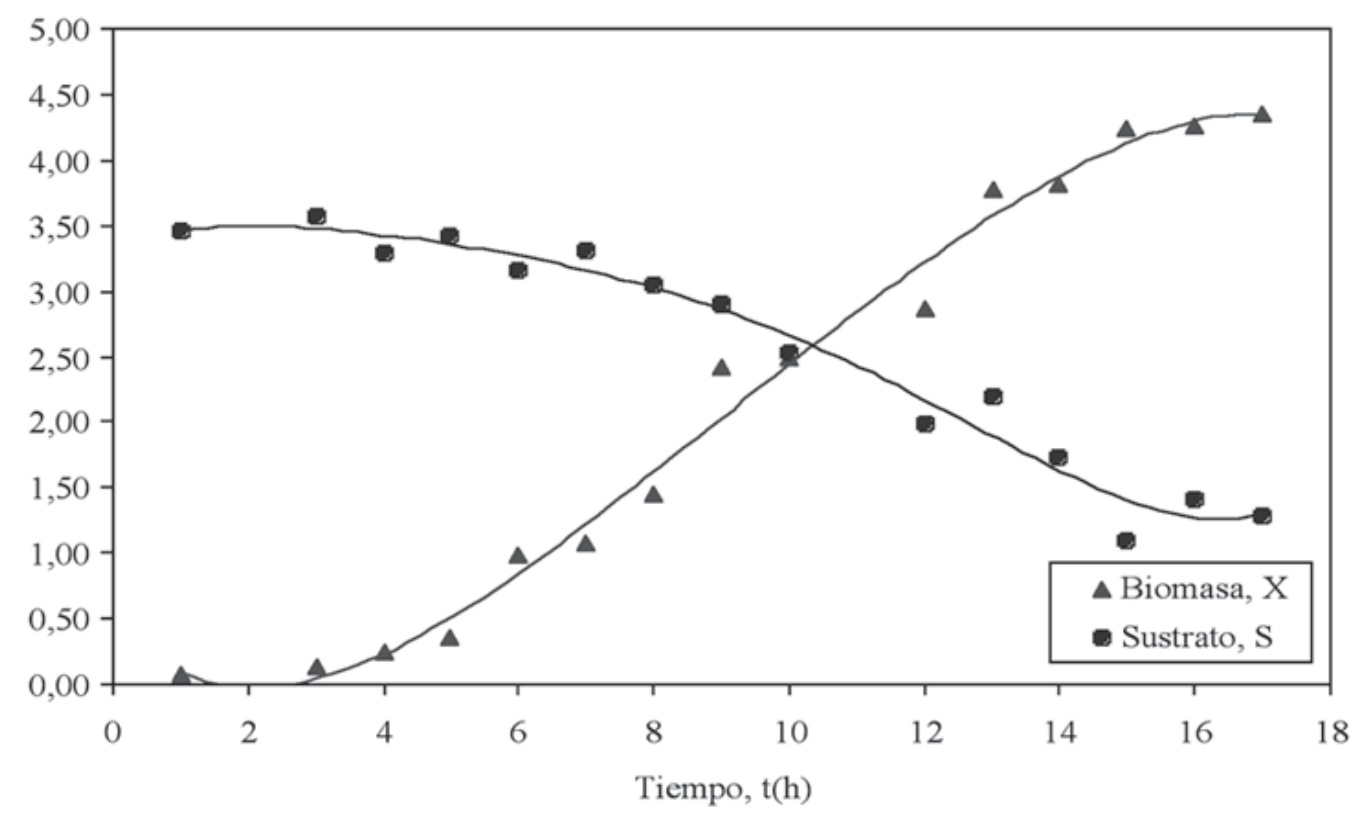

Figura 3. Corrida experimental del seguimiento cinético de la S. cerevisiae a una concentración inicial de 70 millones por mililitro.

Fuente: (Los autores) 
mantuvo aproximadamente constante durante las primeras horas del experimento, coincidiendo con la fase de letargo, donde la demanda de oxígeno es baja, ya que el proceso de reproducción no se ha iniciado; el porcentaje de oxígeno disuelto en el medio de cultivo se mantuvo en un valor promedio entre $65 \%$ a $70 \%$, debido a la poca demanda del microorganismo, por su baja actividad metabólica.
$0,05 \mathrm{~h}^{-1}$ a $0,5 \mathrm{~h}^{-1}$, encontrándose las levaduras en los valores cercanos al límite superior (14). Además, el valor de la constante de saturación, $K s$, obtenido experimentalmente para la $S$. cerevisae, es semejante al informado de $1,7 \mathrm{~g} / \mathrm{L}$ al utilizar glucosa como sustrato (29).

Al analizar la capacidad del microorganismo como alternativa para reducir la carga contaminante del

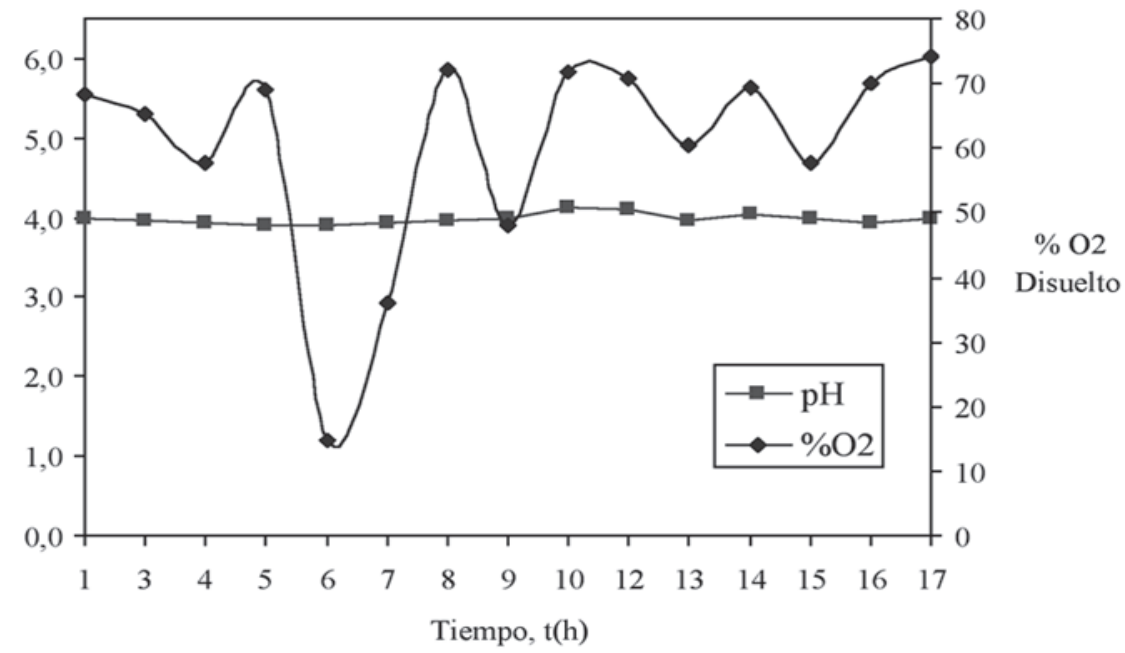

Figura 4. Variación de pH y porcentaje de oxígeno disuelto la corrida experimental del seguimiento cinético con 70 millones de células S. cerevisiae por mililitro.

Fuente: (Los autores)

En la fase de crecimiento exponencial, losrequerimientos de oxígeno por parte del microorganismo comenzaron a aumentar, lo que generó menor cantidad de oxígeno disuelto en el medio de cultivo, como consecuencia de la actividad metabólica; en la fase estacionaria hubo una tendencia más estable en los valores del porcentaje de oxígeno disuelto.

En el Cuadro 5 se presentan los valores para la velocidad específica máxima de crecimiento, $\mu_{\text {máx }}$, y la constante de saturación, $K s$, correspondientes a la segunda corrida experimental.

Se estimó, como criterio general, que el valor de la velocidad específica de crecimiento, para varios microorganismos se encuentra entre agua de coco, se encontró que el DQO inicial fue de $10032 \mathrm{mg} / \mathrm{L}$. Al finalizar el proceso de producción de biomasa, el DQO fue de $5928 \mathrm{mg} / \mathrm{L}$, la diferencia entre ambos valores representó una disminución de un $41 \%$ en la DQO, lo que efectivamente es un avance muy positivo en materia de conservación del medio ambiente contra este tipo de contaminante.

Este resultado permitió considerar los procesos de producción de biomasa, aparte de la posibilidad de producir etanol, como una alternativa para la reducción del impacto ambiental que se genera por la contaminación de éste y otros tipos de sustancias desechadas industrialmente, que son aptas para ser empleadas como sustratos. 
Cuadro 5. Parámetros cinéticos a partir de la curva de crecimiento microbiano obtenida en la segunda corrida experimental

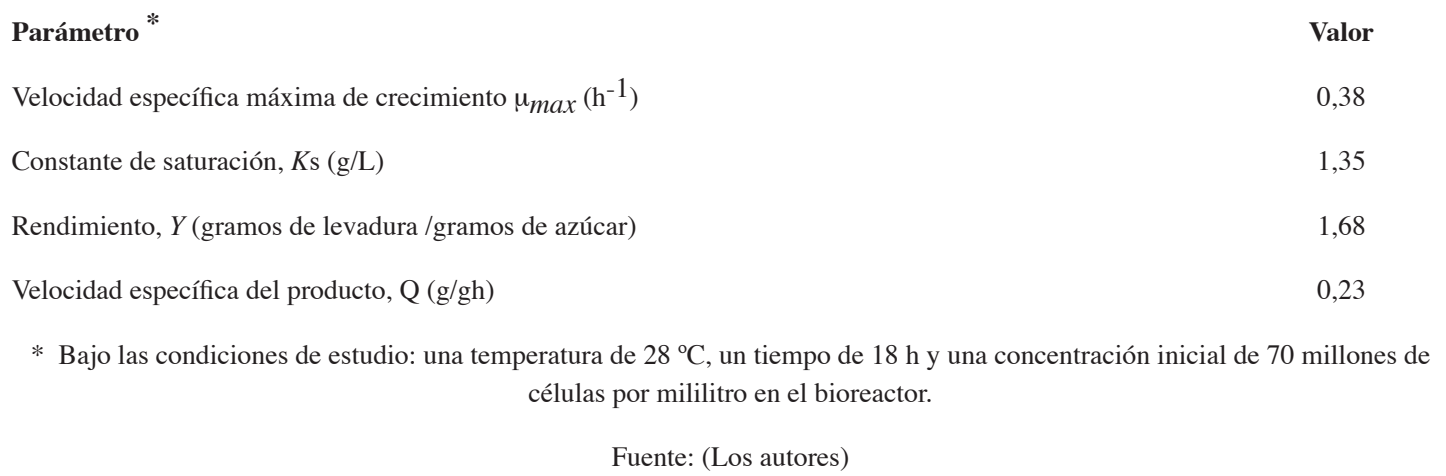

\subsection{Proceso continuo}

La producción de biomasa, en el proceso continuo, se efectuó con las condiciones de cultivo descritas en la metodología. Se realizaron nueve corridas experimentales, a diferentes tasas de dilución y utilizando medio de cultivo fresco en cada experimento.

Se determinaron las concentraciones de biomasa y azúcares totales, tomando muestras del medio de cultivo en cada experimento, hasta obtener las correspondientes al estado estacionario.

En la Figura 5 se aprecia la disminución en la concentración de biomasa conforme aumenta el valor de la tasa de dilución, pasando de un valor inicial de 3,3 g/L para una tasa de dilución de cero, que corresponde a una operación del sistema en forma "batch", hasta una concentración de 0,2 $\mathrm{g} / \mathrm{L}$ para una tasa de dilución igual a $0,5 \mathrm{~h}^{-1}$.

Para la última tasa de dilución $\left(0,5 \mathrm{~h}^{-1}\right)$, la concentración de azúcares totales en el medio de cultivo se mantuvo aproximadamente igual a la concentración del medio de cultivo fresco con que se alimenta el proceso, indicando que la operación del sistema continuo tuvo lugar con una tasa de dilución lo suficientemente alta que provocó el lavado de células o producto, lo cual se debe evitar.

La tasa máxima de dilución, $D_{\max }$, con un valor de $0,19 \mathrm{~h}^{-1}$, se obtuvo gráficamente donde las curvas de concentración de biomasa y sustrato en la Figura 5, se interceptan. De igual forma, la tasa de dilución crítica, $D_{c r i}$, corresponde al valor aproximado de $0,30 \mathrm{~h}^{-1}$, este valor tiene lugar donde la concentración de azúcares totales en el medio de cultivo comienza a mantener un valor constante y similar a la concentración de azúcares totales del medio de cultivo fresco, además, se da inicio al lavado de células. Ambos parámetros son posibles de conocer a partir de los resultados cinéticos obtenidos previamente, estos se muestran conjuntamente en el Cuadro 6.

El valor de la tasa de dilución máxima, obtenida en ambos casos, difiere en un $12 \%$; a valores mayores de $0,30 \mathrm{~h}^{-1}$ da inicio al lavado de células del medio de cultivo.

\section{CONCLUSIONES}

1. Con las variables y los niveles estudiados para cada variable, el flujo de aire es el único que presenta un efecto significativo sobre el rendimiento de producción de biomasa.

2. Del seguimiento cinético se obtuvo un $\mu_{\max }$ de $0,38 \mathrm{~h}^{-1}, K s$ de $1,35 \mathrm{~g} / \mathrm{L}$, un rendimiento (Y) de 1,68 g de levadura/g de azúcar y una velocidad específica de producto $(\mathrm{Q})$ de 0,23 $\mathrm{g} / \mathrm{g} \cdot \mathrm{h}$.

3. La producción de biomasa es una vía factible para los procesos industriales y una alternativa para la reducción de las 


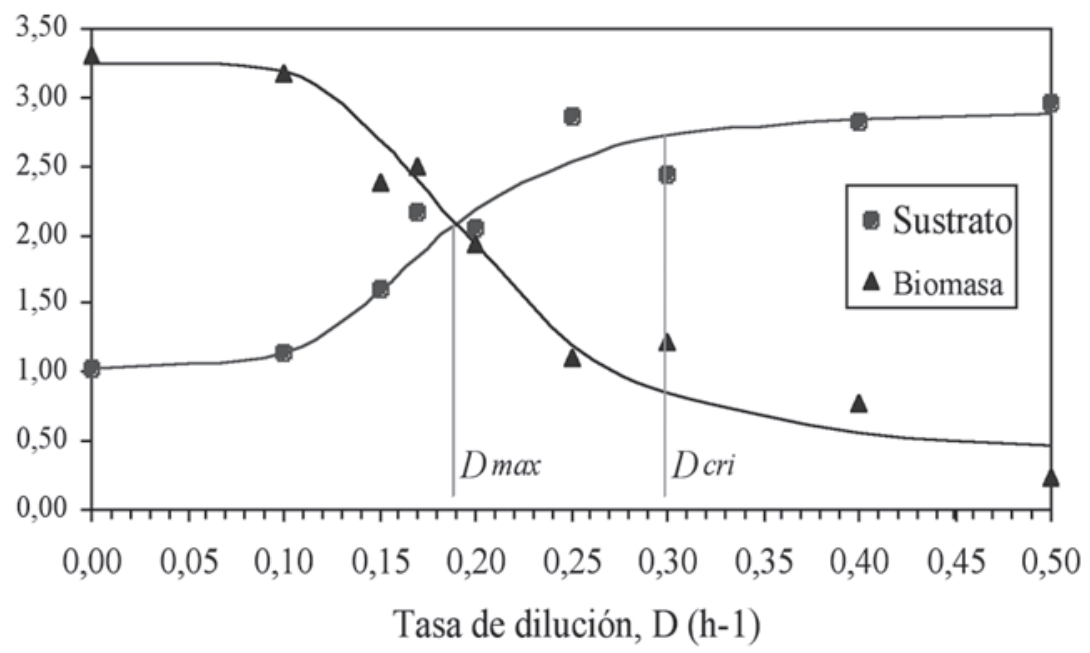

Figura 5. Producción de biomasa en un proceso continuo

Cuadro 6. Parámetros de cultivo continuo*

\begin{tabular}{|c|c|c|}
\hline $\begin{array}{l}\text { Parámetro } \\
\text { cinético }\end{array}$ & $\begin{array}{l}\text { Seguimiento } \\
\text { cinético }\end{array}$ & $\begin{array}{c}\text { Cultivo } \\
\text { continuo }\end{array}$ \\
\hline crítica, $\mathrm{D}_{\text {cri }}\left(\mathrm{h}^{-1}\right)$ & 0,26 & 0,30 \\
\hline lución máxima $\mathrm{D}_{\max }\left(\mathrm{h}^{-1}\right)$ & 0,17 & 0,19 \\
\hline
\end{tabular}

Fuente: (Los autores)

descargas contaminantes, lográndose, en este estudio en particular, una disminución de un $41 \%$ en el valor de la DQO.

4. La producción de biomasa en un proceso continuo, con una tasa de dilución mayor al valor de $0,30 \mathrm{~h}^{-1}$, presenta el lavado de células.

5. Para una buena productividad, la tasa de dilución máxima debe estar entre $0,17 \mathrm{~h}^{-1}$ y $0,19 \mathrm{~h}^{-1}$.

\section{REFERENCIAS BIBLIOGRÁFICAS}

Amato, S. P. (1992). Procesos fermentativos. Curso Latinoamericano de síntesis de bioproductos y escalamiento de procesos biotecnológicos. San José, Universidad de Costa Rica y Universidad Nacional.
Fogler, H. S. (2001). Elementos de Ingeniería de las reacciones químicas. ( $3^{\mathrm{a}}$ ed.). México: Prentice Hall.

Galindo, E. (1993). Transferencia de masa y mezclado de procesos biológicos. Cuarto curso avanzado de procesos biotecnológicos: escalamiento de la producción de proteínas recombinantes, Cuernavaca, México.

Jorgensen, A. (1959). Microbiología de las fermentaciones industriales. ( $7^{\mathrm{a}}$ ed.). Zaragoza: Editorial Acribia.

Metcalf, L. \& Eddy H., (1995). Ingeniería de aguas residuales: tratamiento, vertido y reutilización, $3^{\text {a }}$ ed. Madrid: McGraw Hill.

Monroy, O. (1981). Biotecnología para el aprovechamiento de los desperdicios orgánicos. México: AGT Editor S. A.

Palasi, V. (1952). Microbiología industrial. ( $2^{\mathrm{a}}$ ed.). Madrid: Ediciones Aguilar.

Prescott, S. C. \& Dunns, C. G. (1983). Industrial microbiolog. (4 ${ }^{\text {th }}$ ed.). USA: The Avi Publishing Company Inc.. 
Quintero, R. (1981). Ingeniería bioquímica. (1 ${ }^{\mathrm{a}}$ ed.) México: Alhambra Mexicana.

Ramírez, R. (1994). Modelización matemática $e$ identificación de parámetros en la producción de biomasa para un reactor por tanda. Tesis para optar al grado de Licenciatura en Ingeniería Química, Escuela de Ingeniería Química, Facultad de Ingeniería, Universidad de Costa Rica, San José, Costa Rica.

Rodríguez, B. (1987). Producción de levaduras (Sacharomyces cerevisiae) usando una columna de burbujeo. Tesis para optar por elgrado de Licenciatura en Ingeniería Química, Escuela de Ingeniería Química, Facultad de Ingeniería, Universidad de Costa Rica, San José, Costa Rica.

Stanier, R. Y. (1986). Microbiología. (4a ed.). México: Ediciones Replá S.A..

Vargas, J. (1992). Formulación de un producto a partir de coco, mediante la mezcla de cuatro variedades. Tesis para optar por el grado de Licenciatura en Ingeniería Química, Escuela de Ingeniería Química, Facultad de
Ingeniería, Universidad de Costa Rica, San José, Costa Rica.

Vong Tsoi, A. M. (1996). Estudio de la producción de etanol mediante la fermentación de tres sustratos diferentes obtenidos a partir del banano. Tesis para optar al grado de Licenciatura en Ingeniería Química, Escuela de Ingeniería Química, Facultad de Ingeniería, Universidad de Costa Rica, San José, Costa Rica.

\section{SOBRE LOS AUTORES}

\section{Manuel Molina Córdoba}

Ingeniero Químico

Docente e Investigador de la Universidad de Costa Rica

Teléfonos: 207-46-20/285-34-86

Correo electrónico: emolina@terraba.ing.ucr.ac.cr

\section{Oscar Ramírez Sánchez}

Ingeniero Químico

Tratamiento de Agua Potable en Acueductos y Alcantarillados (A y A)

Teléfonos: 207-46-20/285-34-86

Correo electrónico: oramirez@aya.go.cr 